\title{
A KEPLERIAN-LIKE DISK AROUND THE FORMING O-TYPE STAR AFGL 4176
}

\author{
Katharine G. Johnston ${ }^{1,2}$, Thomas P. Robitaille ${ }^{2}$, Henrik Beuther ${ }^{2}$, Hendrik Linz ${ }^{2}$ Paul Boley $^{3}$, \\ Rolf Kuiper $^{2,4}$, Eric Keto ${ }^{5}$, Melvin G. Hoare ${ }^{1}$, and Roy van Boekel ${ }^{2}$ \\ ${ }^{1}$ School of Physics \& Astronomy, E.C. Stoner Building, The University of Leeds, Leeds LS2 9JT, UK; k.g.johnston@leeds.ac.uk \\ ${ }^{2}$ Max Planck Institute for Astronomy, Königstuhl 17, D-69117 Heidelberg, Germany \\ ${ }^{3}$ Ural Federal University, Astronomical Observatory, 51 pr. Lenina, Ekaterinburg, Russia \\ ${ }^{4}$ Institute of Astronomy and Astrophysics, Eberhard Karls University Tübingen, Auf der Morgenstelle 10, D-72076 Tübingen, Germany \\ ${ }^{5}$ Harvard-Smithsonian Center for Astrophysics, 60 Garden Street, Cambridge, MA 02138, USA \\ Received 2015 September 11; accepted 2015 September 28; published 2015 October 29
}

\begin{abstract}
We present Atacama Large Millimeter/submillimeter Array line and continuum observations at $1.2 \mathrm{~mm}$ with $\sim 0$ !' 3 resolution that uncover a Keplerian-like disk around the forming O-type star AFGL 4176. The continuum emission from the disk at $1.21 \mathrm{~mm}$ (source $\mathrm{mm} 1$ ) has a deconvolved size of $870 \pm 110 \mathrm{AU} \times 330 \pm 300 \mathrm{AU}$ and arises from a structure $\sim 8 M_{\odot}$ in mass, calculated assuming a dust temperature of $190 \mathrm{~K}$. The first-moment maps, pixel-topixel line modeling, assuming local thermodynamic equilibrium (LTE), and position-velocity diagrams of the $\mathrm{CH}_{3} \mathrm{CN} J=13-12 \mathrm{~K}$-line emission all show a velocity gradient along the major axis of the source, coupled with an increase in velocity at small radii, consistent with Keplerian-like rotation. The LTE line modeling shows that where $\mathrm{CH}_{3} \mathrm{CN} J=13-12$ is excited, the temperatures in the disk range from $\sim 70$ to at least $300 \mathrm{~K}$ and that the $\mathrm{H}_{2}$ column density peaks at $2.8 \times 10^{24} \mathrm{~cm}^{-2}$. In addition, we present Atacama Pathfinder Experiment ${ }^{12} \mathrm{CO}$ observations that show a large-scale outflow from AFGL 4176 perpendicular to the major axis of mm1, supporting the disk interpretation. Finally, we present a radiative transfer model of a Keplerian disk surrounding an $\mathrm{O} 7$ star, with a disk mass and radius of $12 M_{\odot}$ and $2000 \mathrm{AU}$ that reproduces the line and continuum data, further supporting our conclusion that our observations have uncovered a Keplerian-like disk around an O-type star.
\end{abstract}

Key words: circumstellar matter - ISM: jets and outflows - radiative transfer - stars: formation - stars: massive techniques: interferometric

\section{INTRODUCTION}

The process of star formation is often pictured as a young star being fed by a disk formed as a result of angular momentum conservation over a period of tens of thousands to millions of years. Yet, this picture has been mainly derived from observations of disks that have practically finished accreting. In actuality, it has been confirmed only relatively recently that stable, rotationally supported, Keplerian disks around low-mass Class 0/I protostars exist at earlier times, when the star and its disk are concealed deeply in an accreting envelope (e.g., Brinch et al. 2007; Jørgensen et al. 2009; Tobin et al. 2012; Murillo et al. 2013).

The situation is even less clear when it comes to forming massive stars, which go on to reach a final mass of $8 M_{\odot}$ and above. As they form so quickly ( $t_{\text {form }} \sim 10^{4}-10^{5}$ years), they spend the entirety of their formation still embedded in a surrounding envelope. Are these protostars the high-mass equivalents of low-mass Class 0/I objects, and do they also have disks? Many of the energetic feedback mechanisms associated with massive stars, such as radiation pressure and at later times stellar winds and photoionization, would halt the vast amount of accretion required to form these stars. In theory, these can be bypassed by the existence of a disk (e.g., Yorke \& Sonnhalter 2002; Krumholz et al. 2009; Kuiper et al. 2010, 2011), allowing the radiation pressure, winds, or hot ionized gas to be channeled away along the axis perpendicular to the disk, where densities are lower.

Recent observations of early B-type (proto)stars have responded to this prediction with detections of disk candidates that have kinematics that appear to be dominated by the central protostar and are stable (e.g., Johnston et al. 2011; Sánchez-
Monge et al. 2013; Beltrán et al. 2014; Cesaroni et al. 2014). Yet, the number of disks discovered so far only constitutes a handful (Cesaroni et al. 2007; Beltrán et al. 2011), as many of the observed rotating structures, instead referred to as toroids, are too large and rotate too slowly to be in centrifugal equilibrium. Stepping up to O-type stars, there are even fewer candidates (e.g., Wang et al. 2012; Hunter et al. 2014; Zapata et al. 2015); in these cases, the detections are based on a velocity gradient across the source and an outflow or jet that is projected perpendicular to the candidate disk plane.

In this Letter, we present Atacama Large Millimeter/ submillimeter Array (ALMA) observations that trace a Keplerian-like disk toward the infrared source AFGL 4176, which constitutes the best observational example of an O-type protostar with a disk to date.

AFGL 4176 (G308.918+0.123, IRAS 13395-6153) is a forming massive star with coordinates $13^{\mathrm{h}} 43^{\mathrm{m}} 01^{\mathrm{s}} 69-62^{\circ} 08^{\prime}$ 51!"3 (FK5 J2000) embedded in a star-forming region with a total luminosity of $\sim 10^{5} L_{\odot}(d=4.2 \mathrm{kpc}$; Green \& McClureGriffiths 2011; Boley et al. 2012). The source lies at the northern edge of an $\mathrm{H}$ II region (Caswell et al. 1992; Ellingsen et al. 2005; Shabala et al. 2006) that peaks $\sim 4^{\prime \prime}$ to the south of AFGL 4176, likely powered by another star of spectral type O9. Four $6.7 \mathrm{GHz}$ Class II methanol maser spots with an extent of $840 \mathrm{AU}$ at $4.2 \mathrm{kpc}$ lie in close proximity to AFGL 4176 along a line with position angle $(\mathrm{PA}) \sim-35^{\circ}$ (Phillips et al. 1998). $\mathrm{NH}_{3}$ observations have uncovered that the star is embedded in a large-scale rotating toroid with a radius of $\sim 0.7 \mathrm{pc}$ (Johnston et al. 2014). The large-scale continuum emission at $1.2 \mathrm{~mm}$ (Beltrán et al. 2006) traces a dense core of $0.8 \mathrm{pc}$ and $890 M_{\odot}$ at $4.2 \mathrm{kpc}$, and knots of shocked $\mathrm{H}_{2}$ emission have been detected (De Buizer et al. 2009), 
suggesting the presence of an outflow. Boley et al. (2012) have modeled the spectral energy distribution and mid-IR interferometric observations of AFGL 4176, finding that the latter required a non-spherically symmetric model to adequately fit the data. They interpreted the mid-IR visibilities as a combination of a disk-like structure with radius of $660 \mathrm{AU}$ at $4.2 \mathrm{kpc}$, inclination of $60^{\circ}$, and $\mathrm{PA}=112^{\circ}$ and a spherically symmetric Gaussian halo with an FWHM of $~ 600$ AU (Boley et al. 2012, 2013). Finally, Ilee et al. (2013) found that the $2.3 \mu \mathrm{m}$ CO bandhead emission toward AFGL 4176 is consistent with the inner $\sim 10$ AU of a Keplerian disk.

\section{OBSERVATIONS}

\subsection{ALMA Observations}

We observed AFGL 4176 with the $12 \mathrm{~m}$ antenna array of ALMA during Cycle 1, under program 2012.1.00469.S (PI: Johnston). The observations were carried out on 2014 August 16 and 17 in dual-polarization mode in Band $6(\sim 250 \mathrm{GHz}$ or $1.2 \mathrm{~mm}$ ) under good weather conditions (precipitable water vapor, $\mathrm{PWV} \sim 1.32$ and $1.14 \mathrm{~mm}$, respectively). AFGL 4176 was observed with one pointing centered on $13^{\mathrm{h}} 43^{\mathrm{m}} 01^{\mathrm{s}} .08$ $-62^{\circ} 08^{\prime} 55^{\prime \prime} 5$ (FK5 J2000). Two wide and two narrow spectral windows (spws) were observed with respective widths of $1.875 \mathrm{GHz}$ and $468.750 \mathrm{MHz}$. The two wide spws were centered at 240.541 and $254.043 \mathrm{GHz}$, while the two narrow spws were centered at frequencies of 239.072 and $256.349 \mathrm{GHz}$. The spectral resolution was $1129 \mathrm{kHz}(1.41$ and $\left.1.33 \mathrm{~km} \mathrm{~s}^{-1}\right)$ and $282 \mathrm{kHz}\left(0.354\right.$ and $\left.0.330 \mathrm{~km} \mathrm{~s}^{-1}\right)$, respectively. Thirty-nine antennas were included in the array, of which 36 had useful data. Baseline lengths were 14.4-1210.8 $\mathrm{m}$, providing a largest angular scale of $\sim 18^{\prime \prime}$. The primary beam size was 22!"7-24!" 4 . The bandpass calibrators were J1617-5848 and J1427-4206 and the absolute flux calibrators were Titan and Ceres on August 16 and 17, respectively. Phase/gain calibrators were J1308-6707 and J1329-5608 for both days. The flux calibration uncertainty was estimated to be $\lesssim 20 \%$.

Calibration was carried out using the Common Astronomy Software Applications (CASA) version 4.2.1 via the delivered pipeline script. We improved the resulting images by selfcalibration of the continuum and applied these solutions to the line data. The continuum images were made using $1.4 \mathrm{GHz}$ bandwidth of line-free channels across all spws. The central frequency of the combined continuum emission is $247.689 \mathrm{GHz}$ $(1.210 \mathrm{~mm})$. Imaging was carried out using Briggs weighting with a robust parameter of 0.5 . The noise in the continuum image is $78 \mu \mathrm{Jy} \mathrm{beam}^{-1}$ in a beam of $0 . .28 \times 0$.! 24 , $\mathrm{PA}=-30^{\circ}$.2. In this Letter, we also present the detected $K$ ladder transitions of $\mathrm{CH}_{3} \mathrm{CN} J=13-12$. The $K=0-8$ transitions were observed within the $239.072 \mathrm{GHz}$ band. For the $K=2-8$ images presented below, the noise ranges between 3.4 and $6.2 \mathrm{mJy}^{\text {beam }^{-1}}$ in a beam of 0 . $30 \times 0$." $28, \mathrm{PA}=37^{\circ} .7-37^{\circ} .9$.

\subsection{Atacama Pathfinder Experiment (APEX) Observations}

We observed AFGL 4176 with the APEX 612 m antenna for program M0020_89 during the night of 2012 April 20-21 under very good weather (PWV $\sim 0.4 \mathrm{~mm}$ ). The Swedish

\footnotetext{
6 APEX is a collaboration between the Max-Planck-Institut für Radioastronomie, the European Southern Observatory, and the Onsala Space Observatory.
}

Heterodyne Facility Instrument APEX-2 receiver was tuned to ${ }^{12} \mathrm{CO}(3-2)$ at $345.79599 \mathrm{GHz}$ in the lower sideband, providing a beam size of $18^{\prime \prime}$. On-the-fly maps with an extent of 2 . $5 \times 2$ '.5 were taken in two perpendicular scan directions to reduce scanning artifacts. The XFFTS2 backend provided a channel separation of $0.1984 \mathrm{~km} \mathrm{~s}^{-1}$. The achieved $\mathrm{rms}$ in the spectra in the central part of the map is around $0.12 \mathrm{~K}$. The data reduction was performed within GILDAS/CLASS. ${ }^{7}$ The units of the spectral cube are in corrected antenna temperatures $\left(T_{\mathrm{A}}^{*}\right)$.

\section{RESULTS AND DISCUSSION}

In Figure 1, we present the $1.21 \mathrm{~mm}$ continuum emission from the AFGL 4176 region. The emission is dominated by $\mathrm{mm} 1$, with a peak position in the non-self-calibrated image of $13^{\mathrm{h}} 43^{\mathrm{m}} 01.693$ $-62^{\circ} 08^{\prime} 51$ !' 25 (FK5 J2000), coincident with the position of AFGL 4176 in 2 MASS (with $\sim 0$ "! 1 positional uncertainty ${ }^{8}$ ). Performing a Gaussian fit to mm1, we determined a peak flux of $37 \pm 2 \mathrm{mJy}$ beam $^{-1}$, an integrated flux of $50 \pm 4 \mathrm{mJy}$, a deconvolved size of 0 ". $21 \pm 0$ ". $03 \times 0$." $08 \pm 0$ ". 07 $(870 \pm 110 \mathrm{AU} \times 330 \pm 300 \mathrm{AU})$, and $\mathrm{PA}=59^{\circ} \pm 17^{\circ}$. Using the equations of Hildebrand (1983), as well as assuming $190 \mathrm{~K}$ (the average temperature of the disk derived from the CASSIS LTE line modeling below), a gas-to-dust ratio of 154 (Draine 2011), and a dust opacity at $1.21 \mathrm{~mm}$ of $0.24 \mathrm{~cm}^{2} \mathrm{~g}^{-1}$ (Draine 2003a, 2003b, with $R_{V}=5.5$ ), we derive a gas mass and peak column density of $8 M_{\odot}$ and $8 \times 10^{24} \mathrm{~cm}^{-2}$ for mm1. As well as a compact component, mm1 includes low-lying $5 \sigma-10 \sigma$ emission extending NW, perpendicular to the major axis of $\mathrm{mm} 1$, that joins it to $\mathrm{mm} 2$. The remaining millimeter sources lie in the NW and SE quadrants around $\mathrm{mm} 1$, including two compact sources, $\mathrm{mm} 2$ and $\mathrm{mm} 4$, on opposite sides of $\mathrm{mm} 1$. Full observed properties of all detected $1.21 \mathrm{~mm}$ sources will be given in a upcoming paper (K. G. Johnston et al. 2016, in preparation).

Figure 2 shows the integrated ${ }^{12} \mathrm{CO} J=3-2$ emission from the red- and blueshifted high-velocity wings of the bipolar outflow from AFGL 4176 observed by APEX, overlaid upon a ${ }^{12} \mathrm{CO}$ zero-moment map shown in grayscale. The outflow lobes are orientated roughly NW-SE, perpendicular to the PA of the source $\mathrm{mm} 1$, shown as a dashed line in Figure 2. This relative geometry suggests $\mathrm{mm} 1$ is a disk driving the large-scale outflow seen in ${ }^{12} \mathrm{CO}$.

The kinematics of the gas in $\mathrm{mm} 1$ traced by first- and second-moment maps (intensity-weighted velocity and linewidth fields, respectively) of $\mathrm{CH}_{3} \mathrm{CN} J=13-12 \mathrm{~K}=3$ emission are shown in panels (a) and (b) of Figure 3. The velocity field of the $K=3$ line in panel (a) shows a clear velocity gradient along the major axis of $\mathrm{mm} 1$, which is also present in lines $K=2-8$ and on arcminute scales in $\mathrm{NH}_{3}$ (Johnston et al. 2014). The velocity field shown in Figure 3 is similar to that expected from disks in near-Keplerian rotation (e.g., HD 100546 and TW Hya, respectively; Hughes et al. 2011; Pineda et al. 2014). For instance, there is a quick change from blue- to redshifted emission when crossing the minor axis of the source. In addition, the emission in the most blue- and redshifted channels is found close to the continuum peak position, whereas the lower-velocity gas is more extended. The linewidths across $\mathrm{mm} 1$ in panel (b) peak toward the continuum

\footnotetext{
http://www.iram.fr/IRAMFR/GILDAS

8 http://www.ipac.caltech.edu/2mass/releases/allsky/doc/sec1_6b. html\#psrecptsour
} 


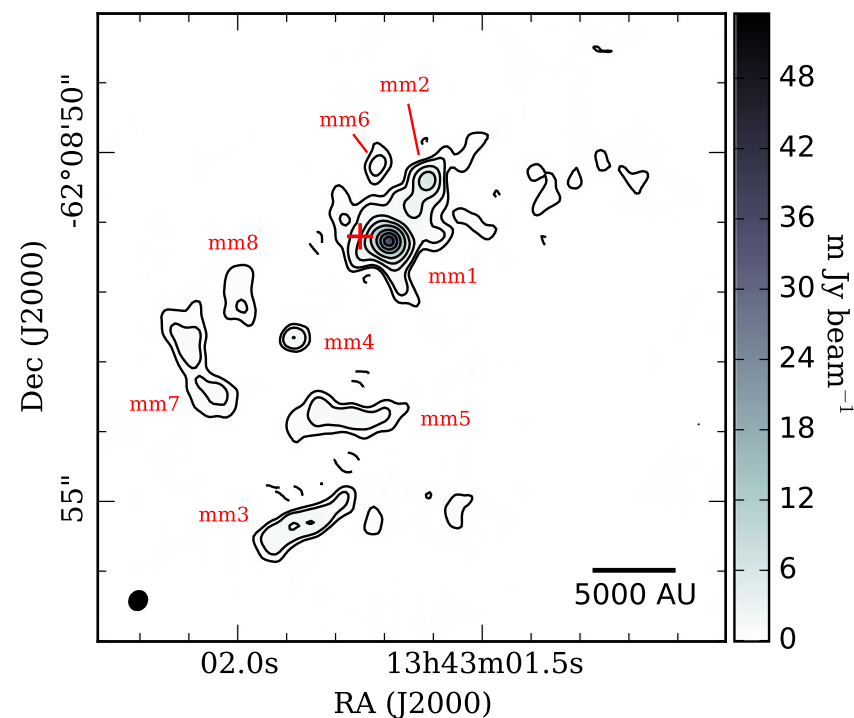

Figure 1. Continuum emission toward AFGL 4176 at $1.21 \mathrm{~mm}$ observed with ALMA, in grayscale and contours $\left(\sigma=78 \mu \mathrm{Jy} \mathrm{beam}^{-1} \times-5,5,10,25,50\right.$, $100,200,300,400)$. The beam is shown in the bottom left corner. The red cross shows the position of the Class II methanol maser group reported in Phillips et al. (1998) and the millimeter sources are labeled.

peak, consistent with beam-averaging of high-velocity red- and blueshifted emission. There is extended $\mathrm{CH}_{3} \mathrm{CN}$ emission to the NW of $\mathrm{mm} 1$ with near-systemic velocities $\left(v_{\mathrm{LSR}} \sim\right.$ $-52 \mathrm{~km} \mathrm{~s}^{-1}$ ), as well as blueshifted gas associated with $\mathrm{mm} 2$, which would be consistent with this source being associated with the blueshifted lobe seen in ${ }^{12} \mathrm{CO}$.

Figures 3(c)-(f) present the results of modeling the $\mathrm{CH}_{3} \mathrm{CN}$ and $\mathrm{CH}_{3}^{13} \mathrm{CN} J=13-12 \mathrm{~K}$-ladders in the spectrum associated with each pixel, making a map of excitation temperature $\left(T_{\mathrm{ex}}\right)$, velocity $\left(v_{\text {LSR }}\right)$, linewidth $\left(v_{\text {FWHM }}\right)$, and column density $\left(N_{\text {mol }}\right)$ across the source. The modeling was carried out using CASSIS $^{9}$ and the JPL molecular spectroscopy database ${ }^{10}$ using the Markov Chain Monte Carlo $\chi^{2}$ minimization method and assuming local thermodynamic equilibrium (LTE). Given $N_{\text {mol }}$ and $T_{\mathrm{ex}}$, CASSIS determines an optical depth that is included in each model. We assume a $\left[{ }^{12} \mathrm{C} /{ }^{13} \mathrm{C}\right]$ abundance ratio of 60 (assuming the distance to the Galactic center is $8.4 \mathrm{kpc}$ and thus a Galactocentric distance to AFGL 4176 of $6.6 \mathrm{kpc}$; Milam et al. 2005; Reid et al. 2009), a source size of 0."53 from the fitted size of the $K=0,1$ zero-moment map, and initial parameter ranges $T_{\mathrm{ex}}=50-350 \mathrm{~K}, v_{\mathrm{LSR}}=-57$ to $-47 \mathrm{~km} \mathrm{~s}^{-1}$, $v_{\text {FWHM }}=0.5-10 \mathrm{~km} \mathrm{~s}^{-1}$, and $N_{\mathrm{mol}}=1 \times 10^{14}-1 \times 10^{17} \mathrm{~cm}^{-2}$. The resultant temperature shown in panel (c) peaks at the continuum peak position and has values ranging between 74 and $294 \mathrm{~K}$. There is also a secondary temperature peak toward mm2. Panels (d) and (e) show similar results to the first- and second-moment maps shown in panels (a) and (b); however, an increase in linewidth can be seen more clearly toward $\mathrm{mm} 2$. The column density of $\mathrm{CH}_{3} \mathrm{CN}$ peaks at $2.8 \times 10^{16} \mathrm{~cm}^{-2}$ toward the center of $\mathrm{mm} 1$, with a slight elongation along the major axis. Using the assumed size above and an abundance of $\mathrm{CH}_{3} \mathrm{CN}$ of $10^{-8}$ (e.g., Gibb et al. 2000; Bisschop et al. 2007), this corresponds to $\mathrm{H}_{2}$ column and volume densities of $2.8 \times 10^{24} \mathrm{~cm}^{-2}$ and $>8 \times 10^{7} \mathrm{~cm}^{-3}$, respectively.

\footnotetext{
9 CASSIS is developed by IRAP-UPS/CNRS (http://cassis.irap.omp.eu).

${ }^{10} \mathrm{http}: / /$ spec.jpl.nasa.gov
}

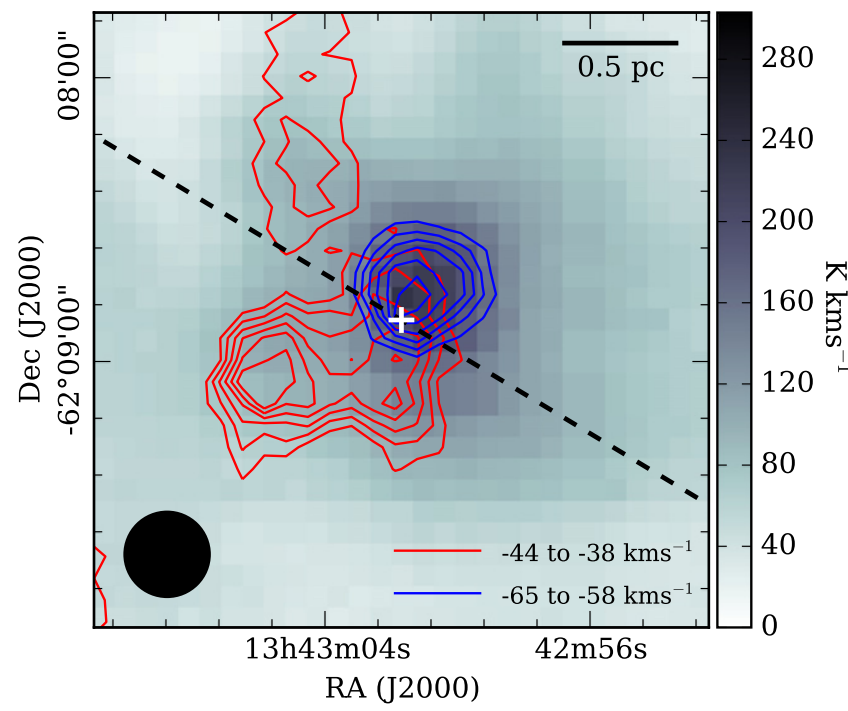

Figure 2. Large-scale bipolar outflow from AFGL 4176 seen in ${ }^{12} \mathrm{CO} J=3-2$ with APEX. The total integrated emission is shown in grayscale, and the integrated red- and blueshifted emission is shown in contours at $40 \%, 50 \%$, $60 \%, \ldots, 90 \%$ of the peak integrated fluxes $\left(11.5\right.$ and $24.9 \mathrm{~K} \mathrm{~km} \mathrm{~s}^{-1}$, respectively). Velocity ranges for the integration of the red- and blueshifted emission are shown in the bottom right, and the beam in the bottom left. The cross and dashed line mark the peak and PA of the continuum emission shown in Figure 1.

Having established that a rotating structure, likely a disk, surrounds AFGL $4176 \mathrm{~mm}$, we can compare it with the geometry of the circumstellar material determined from modeling observations from the MID-infrared interferometric Instrument (MIDI; Boley et al. 2012, 2013). The PA of $\mathrm{mm} 1\left(\sim 60^{\circ}\right)$ is not aligned with the $112^{\circ}$ determined from the MIDI visibilities. Although these PA are not exactly orthogonal, these two observations may be reconciled if the MIDI observations are instead tracing heated dust in the outflow cavity, such as found for W33 A (de Wit et al. 2010). We also note that although offset from the continuum peak of $\mathrm{mm} 1$, the four Class II methanol maser spots detected by Phillips et al. (1998), shown in Figure 3 (f), lie along a line almost perpendicular to the disk.

To investigate the dynamics of $\mathrm{mm} 1$ further, we present position-velocity (PV) diagrams of the $\mathrm{CH}_{3} \mathrm{CN} J=13-12 \mathrm{~K}=2$, 4, 6, and 8 lines in Figures 4(a)-(d) along a cut centered on the continuum peak position of $\mathrm{mm} 1$, with width $=1^{\prime \prime}$ and $\mathrm{PA}=61.5$, which was the average PA of the emission in the $\mathrm{CH}_{3} \mathrm{CN} \mathrm{J}=13-12 \mathrm{~K}=4,5$, and 6 lines that had good signal-tonoise and were not contaminated by envelope emission.

The PV diagrams of the $K=2,4$, and 6 lines exhibit the "butterfly" shape expected from a Keplerian-like rotation curve that increases in velocity close to the central object and is more extended close to the systemic velocity. It bears close resemblance to the PV diagrams observed toward the forming early B-type star IRAS $20126+4014$, which has a disk in Keplerian-like rotation (Cesaroni et al. 2005, 2014), as well as the forming O-type stars NGC 6334 I(N) SMA 1b and IRAS 16547-4247 (Hunter et al. 2014 and Zapata et al. 2015, respectively). The velocity gradient of the $K$-lines becomes steeper at higher values of $K$, thus the more-excited lines that trace hotter gas closer to the central object also trace higher velocities, which is expected from Keplerian-like rotation.

Interestingly, the PV diagrams of the lower $K$-lines shown in Figure 4 are asymmetrical about the systemic velocity, with the blueshifted emission being brighter. This is not observed 

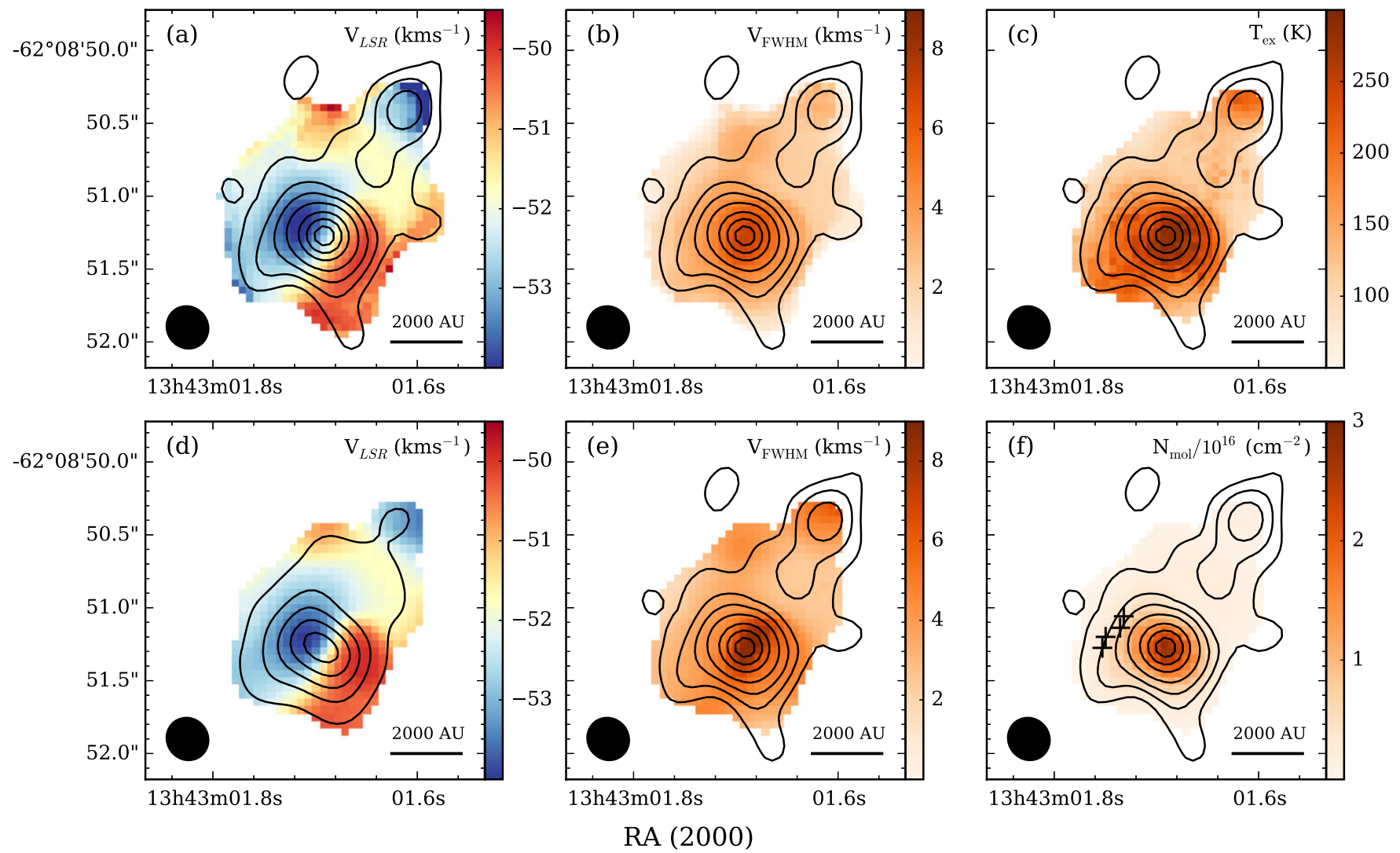

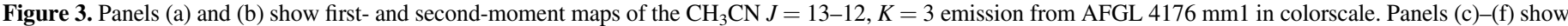

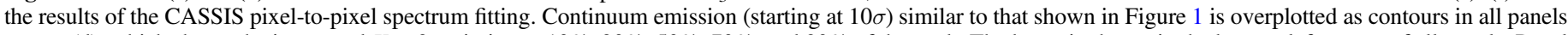

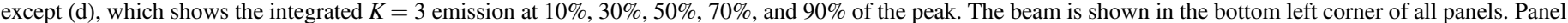
(f) shows the positions of the Class II methanol masers from Phillips et al. (1998).

however in the $K=7,8$ lines. A similar blue asymmetry is seen in the PV diagrams of the low-excitation $K$-lines of $\mathrm{CH}_{3} \mathrm{CN}$ $J=12-11$ observed by Cesaroni et al. (2014) and Hunter et al. (2014), whereas the high-excitation lines are either symmetrical or exhibit a red asymmetry. The $\mathrm{CH}_{3} \mathrm{CN} J=19-18 K=2 \mathrm{PV}$ diagram of $\mathrm{G} 35.03+0.35 \mathrm{HMCA}$ also shows this blue asymmetry (Beltrán et al. 2014). This has been suggested to be due to an asymmetric disk structure; however, the fact that so many objects exhibit the same features in their PV diagrams suggests this is more likely due to a radiative transfer effect and/or a geometry that is present in all sources.

To check whether a Keplerian-disk model is consistent with our observations, we ran a grid of self-consistent gas and dust radiative transfer models, where the line and continuum radiative transfer were performed using the codes MolliE (assuming LTE; Keto \& Caselli 2010) and HyPERION (Robitaille 2011), using the Milky Way dust properties from Draine (2003a, 2003b) with $R_{V}=5.5$, respectively. To fit the models to the line and continuum observations, we fit the profiles of the continuum and $\mathrm{CH}_{3} \mathrm{CN} J=13-12 \mathrm{~K}=2-8$ emission collapsed along the major and minor axes, as well as the integrated spectra for the lines. The models were convolved to the observed beam before fitting.

Panels (e)-(h) of Figure 4 show the $\mathrm{CH}_{3} \mathrm{CN} J=13-12 \mathrm{PV}$ diagrams for a model that provides a good fit to the line and continuum data. The model consists of a Keplerian flared disk of radius $2000 \mathrm{AU}$ (the inner radius is set to be the dust sublimation radius, which in this case is $31.3 \mathrm{AU}$ ), total gas mass $12 M_{\odot}$, with a surface density decreasing as $r^{-1.5}$, and an inclination of $30^{\circ}$. The scale height of the disk is given by $z=6.7(\varpi / 100 \mathrm{AU})^{1.29} \mathrm{AU}$, where $\varpi$ is the cylindrical radius and the constants were determined in order for the disk to be in hydrostatic equilibrium. The model includes a rotationally flattened infalling envelope (Ulrich 1976) with an infall rate of $4.6 \times 10^{-4} M_{\odot} \mathrm{yr}^{-1}$ and an outer radius of $150,000 \mathrm{AU}$. The central object is a $25 M_{\odot}$ zero-age main-sequence O7 star, with properties determined from Meynet \& Maeder (2000) and Martins et al. (2005), specifically $T=36,872 \mathrm{~K}$ and $R=6.71 R_{\odot}$. The Keplerian velocity field in the model accounts for the circumstellar mass interior to a given radius. The abundance of $\mathrm{CH}_{3} \mathrm{CN}$ relative to $\mathrm{H}_{2}$ is taken to be $10^{-8}$ at $>100 \mathrm{~K}$, dropping to $5 \times 10^{-9}$ for $90 \mathrm{~K}<T<100 \mathrm{~K}$, and again to $10^{-10}$ for $<90 \mathrm{~K}$ (Collings et al. 2004; Gerner et al. 2014). The grid of models as well as more detailed results will be presented in a future publication (K. G. Johnston et al. 2016, in preparation).

We note that the model disk mass of $12 M_{\odot}$ differs from that derived using the single temperature of $190 \mathrm{~K}\left(8 M_{\odot}\right)$ because it accounts for the expected variance in temperature and optical depth over the disk and is the best fit from a discrete set of disk masses. In addition, a Gaussian fit to the continuum model image gives a major FWHM of $880 \mathrm{AU}$, in good agreement with the fit to the observations (FWHM $=870 \mathrm{AU}$ ), which indicates the Gaussian fit underestimates the actual disk size.

\section{CONCLUSIONS}

We present ALMA observations that uncover the presence of a Keplerian-like disk around an O-type star, detected in both $1.21 \mathrm{~mm}$ continuum and $\mathrm{CH}_{3} \mathrm{CN}$. The velocity structure as traced 


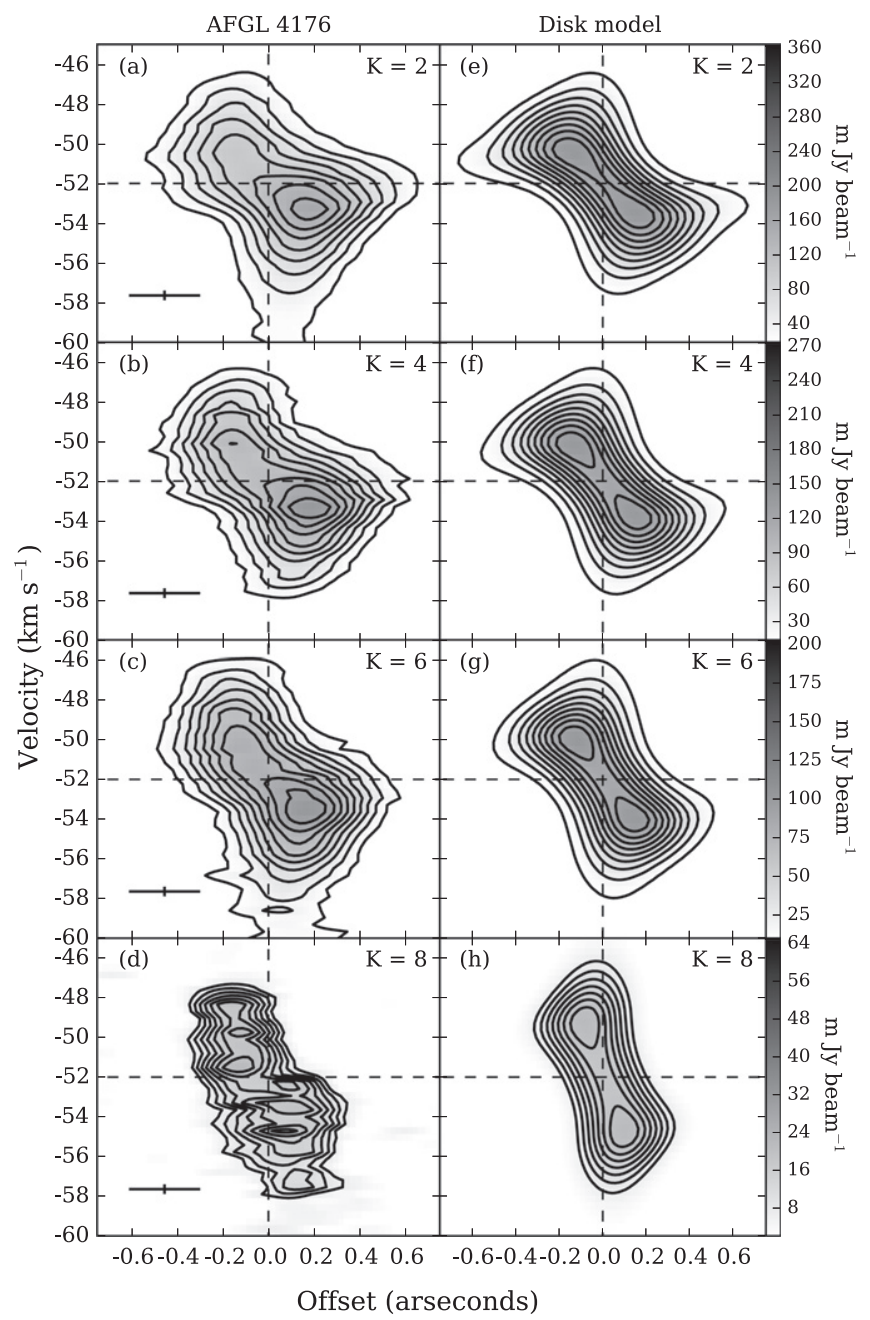

Figure 4. Panels (a)-(d): position-velocity diagrams of $\mathrm{CH}_{3} \mathrm{CN}$ $J=13-12 K=2,4,6$, and 8 averaged along a cut centered on the $\mathrm{mm} 1$ continuum peak position, with $\mathrm{PA}=61.5$ and width $=1^{\prime \prime}$. The horizontal and vertical dashed lines mark the position of the continuum peak and a velocity of $-52 \mathrm{~km} \mathrm{~s}^{-1}$. The crosses in the bottom left of each panel show the observational spatial and spectral resolution. Panels (e)-(h): position-velocity diagrams of the same lines for the model described in the text. Contour levels are $10 \%, 20 \%, 30 \%, \ldots, 90 \%$ of the peak flux, except for $K=8$, which instead starts at $30 \%$.

by $\mathrm{CH}_{3} \mathrm{CN}$ moment maps and LTE line modeling shows a clear velocity gradient and evidence of higher velocities at small radii, as expected for a Keplerian disk. In addition, we present a radiative transfer model that agrees with our ALMA observations not only in terms of the morphology of the PV diagram, but also in terms of the absolute flux of the lines and the continuum, and corroborates that we have uncovered the best example to date of a disk in Keplerian-like rotation around a forming O-type star.

We thank the referee for insightful comments that helped improve this Letter. We are grateful to have been able to observe with ALMA and APEX on Llano de Chajnantor, Chile. ALMA is a partnership of ESO (representing its member states), NSF (USA) and NINS (Japan), together with NRC (Canada), NSC and ASIAA (Taiwan), and KASI (Republic of Korea), in cooperation with the Republic of Chile. The Joint ALMA Observatory is operated by ESO, AUI/NRAO and NAOJ. We thank Frank Wyrowski, Miguel Angel Requena
Torres, and our ALMA contact scientist Edwige Chapillon. P.B. acknowledges support from the Russian Science Foundation, grant No. 15-12-10017.

\section{REFERENCES}

Beltrán, M. T., Brand, J., Cesaroni, R., et al. 2006, A\&A, 447, 221

Beltrán, M. T., Cesaroni, R., Neri, R., \& Codella, C. 2011, A\&A, 525, A151 Beltrán, M. T., Sánchez-Monge, Á., Cesaroni, R., et al. 2014, A\&A, 571, A52 Bisschop, S. E., Jørgensen, J. K., van Dishoeck, E. F., \& de Wachter, E. B. M. 2007, A\&A, 465, 913

Boley, P. A., Linz, H., van Boekel, R., et al. 2012, A\&A, 547, A88

Boley, P. A., Linz, H., van Boekel, R., et al. 2013, A\&A, 557, C1

Brinch, C., Crapsi, A., Jorgensen, J. K., Hogerheijde, M. R., \& Hill, T. 2007 A\&A, 475, 915

Caswell, J. L., Kesteven, M. J., Stewart, R. T., Milne, D. K., \& Haynes, R. F. 1992, ApJL, 399, L151

Cesaroni, R., Galli, D., Lodato, G., Walmsley, C. M., \& Zhang, Q. 2007, in Protostars and Planets V, ed. B. Reipurth, D. Jewitt \& K. Keil (Tuscon, AZ: Univ. Arizona Press), 197

Cesaroni, R., Galli, D., Neri, R., \& Walmsley, C. M. 2014, A\&A, 566, A73

Cesaroni, R., Neri, R., Olmi, L., et al. 2005, A\&A, 434, 1039

Collings, M. P., Anderson, M. A., Chen, R., et al. 2004, MNRAS, 354, 1133

De Buizer, J. M., Redman, R. O., Longmore, S. N., Caswell, J., \& Feldman, P. A. 2009, A\&A, 493, 127

de Wit, W. J., Hoare, M. G., Oudmaijer, R. D., \& Lumsden, S. L. 2010, A\&A, 515, A45

Draine, B. T. 2003a, ARA\&A, 41, 241

Draine, B. T. 2003b, ApJ, 598, 1017

Draine, B. T. 2011, Physics of the Interstellar and Intergalactic Medium (Princeton, NJ: Princeton Univ. Press)

Ellingsen, S. P., Shabala, S. S., \& Kurtz, S. E. 2005, MNRAS, 357, 1003

Gerner, T., Beuther, H., Semenov, D., et al. 2014, A\&A, 563, A97

Gibb, E., Nummelin, A., Irvine, W. M., Whittet, D. C. B., \& Bergman, P. 2000 , ApJ, 545, 309

Green, J. A., \& McClure-Griffiths, N. M. 2011, MNRAS, 417, 2500

Hildebrand, R. H. 1983, QJRAS, 24, 267

Hughes, A. M., Wilner, D. J., Andrews, S. M., Qi, C., \& Hogerheijde, M. R. 2011, ApJ, 727, 85

Hunter, T. R., Brogan, C. L., Cyganowski, C. J., \& Young, K. H. 2014, ApJ, 788,187

Ilee, J. D., Wheelwright, H. E., Oudmaijer, R. D., et al. 2013, MNRAS, 429, 2960

Johnston, K. G., Beuther, H., Linz, H., et al. 2014, in The Labyrinth of Star Formation (Astrophysics and Space Science Proceedings, Vol. 36; Cham, Switzerland: Springer International), 413

Johnston, K. G., Keto, E., Robitaille, T. P., \& Wood, K. 2011, MNRAS, 415,2953

Jørgensen, J. K., van Dishoeck, E. F., Visser, R., et al. 2009, A\&A, 507, 861

Keto, E., \& Caselli, P. 2010, MNRAS, 402, 1625

Krumholz, M. R., Klein, R. I., McKee, C. F., Offner, S. S. R., \& Cunningham, A. J. 2009, Sci, 323, 754

Kuiper, R., Klahr, H., Beuther, H., \& Henning, T. 2010, ApJ, 722, 1556

Kuiper, R., Klahr, H., Beuther, H., \& Henning, T. 2011, ApJ, 732, 20

Martins, F., Schaerer, D., \& Hillier, D. J. 2005, A\&A, 436, 1049

Meynet, G., \& Maeder, A. 2000, A\&A, 361, 101

Milam, S. N., Savage, C., Brewster, M. A., Ziurys, L. M., \& Wyckoff, S. 2005, ApJ, 634, 1126

Murillo, N. M., Lai, S.-P., Bruderer, S., Harsono, D., \& van Dishoeck, E. F. 2013, A\&A, 560, A103

Phillips, C. J., Norris, R. P., Ellingsen, S. P., \& McCulloch, P. M. 1998, MNRAS, 300, 1131

Pineda, J. E., Quanz, S. P., Meru, F., et al. 2014, ApJL, 788, L34

Reid, M. J., Menten, K. M., Zheng, X. W., et al. 2009, ApJ, 700, 137

Robitaille, T. P. 2011, A\&A, 536, A79

Sánchez-Monge, Á., Cesaroni, R., Beltrán, M. T., et al. 2013, A\&A, 552, L10

Shabala, S. S., Ellingsen, S. P., Kurtz, S. E., \& Forbes, L. K. 2006, MNRAS, 372,457

Tobin, J. J., Hartmann, L., Chiang, H.-F., et al. 2012, Natur, 492, 83

Ulrich, R. K. 1976, ApJ, 210, 377

Wang, K.-S., van der Tak, F. F. S., \& Hogerheijde, M. R. 2012, A\&A, 543, A22

Yorke, H. W., \& Sonnhalter, C. 2002, ApJ, 569, 846

Zapata, L. A., Palau, A., Galván-Madrid, R., et al. 2015, MNRAS, 447, 1826 